\title{
A feasibility study of SMC-MIMO based control architecture for longitudinal high angle of attack flight
}

\author{
N Rouyan, Y Chak, R Varatharajoo* \\ Department of Aerospace Engineering, Institute for \\ Mathematical Research (INSPEM), Malaysia-Italy Centre of \\ Excellence for Mathematical Sciences (MICEMS), Universiti \\ Putra Malaysia, Malaysia
}

\begin{abstract}
Aircraft is a highly non-linear vehicle especially when it flies at high angles of attack due to flow separations which cause non-linear aerodynamic characteristics in that region. To deal with the non-linearity of the aircraft in this region, a robust control method for a multi-input multi-output or MIMO problem is considered in this feasibility review. Sliding mode control (SMC) approach was selected as it is one of the robust and nonlinear control methods. In this paper, the controller objective is to track the angles of attack and the pitch angle throughout the high angles of attack envelope itself. Numerical simulations are carried out with respect to high angles of attack maneuver; and the novel integrated SMC-MIMO system performances were studied accordingly based on their transient responses. The results show the feasibility of the proposed control architecture for the high angles of attack within the flight envelopes.
\end{abstract}

\section{INTRODUCTION}

Aircraft dynamics are known to be non-linear especially flying at high angles of attack region. The region is limited by the maximum lift coefficient and is considered dangerous due to drastic changes in the aircraft dynamics that are introduced by the non-linearities in the aerodynamic characteristics. As the aircraft enters this region, it can end up in a critical flight condition such as a stall or a spin. Though the necessity for an aircraft to fly at high angles of attack may be low, but it may be encountered in some situations for examples, avoiding collision with terrains, following evasive manoeuvres, flying during bad weather conditions, reducing landing distance or during some other emergencies.

Conventionally, flight control systems are designed based on approximately linear mathematical models at various flight conditions based on the non-linear model. Then, a controller parameter is varied with the flight conditions in the gain scheduled approach [1], [2]. However, a great amount of assessment is required to provide enough reference at offdesign point and actual system performance and stability can be different from the design results. To overcome such limitations, a number of researchers started to explore non-linear control techniques such as dynamic inversion which forces a non-linear system to behave linearly from a synthetic input to a desired output [3], [4]. It is an advance control method which can directly handle nonlinear system with less gain schedule and provides inherent

${ }^{\star}$ Corresponding Author: renu99@gmx.de 
decoupled property. However, the controller is lacking in robustness and that sometimes aircraft system is considered as a non-minimum phase system. If not much care is taken into consideration, the non-minimum phase condition might lead to unstable internal dynamics [5]. Thus, several papers proposed an integration of dynamic inversion with other robust controllers such as structured singular value $(\mu)$ synthesis [6], [7], quantitative feedback theory [8] and stochastic probabilistic method [9] to deal with uncertainty, which are complex in terms of their computations.

On the other hand, the implementation of sliding mode control (SMC) on a nonlinear aircraft model has promised robustness due to its insensitivity to modelling inaccuracies and reduction in the complexity of feedback design [10]-[12]. In developing the sliding mode control design, a sliding surface is chosen from the output variables. The control action in sliding mode control involves two phases; sliding phase and reaching phase. In sliding phase, the system performance is completely determined by the sliding manifold design, which is insensitive to matched uncertainties. While in reaching phase, the system is forced to reach the desired sliding surface.

This control approach was initiated by Emilyanov in early 1950. Since then, it has been applied to various control problems including spacecraft attitude control [13], air to air missile control [14], [15] and aircraft flight control. Though there are already works dealt with the SMC on aircraft controls with different set of objectives, the SMCs were either designed based on linearization of the aircraft model [11], [12], and implemented for the lateral-directional motion of the aircraft [16]-[18], or designed as a reconfigurable control for a damage aircraft [19].

This feasibility review attempts on applying the SMC for multi-input-multi-output (MIMO) aircraft system, which is the novel highlight herein. The angle of attack $\alpha$ and the pitch angle $\theta$ appear to be correlated and are chosen as the tracking output. The angle of attack is important because if the critical angle of attack is exceeded, then the aircraft will go into in a stall condition.

\section{MATHEMATHICAL MODEL OF AIRCRAFT}

The aircraft model used in this work is based on the F-18 fighter aircraft [20]. The longitudinal dynamics motion for the aircraft are given by [21]:

$$
\begin{gathered}
\dot{V}=\frac{1}{m}\left(-\bar{q} S C_{D}+2 T \cos \alpha \cos 1.98^{\circ}-m g \sin \theta \cos \alpha\right) \\
\dot{\alpha}=\frac{1}{m V}\left(-\bar{q} S C_{L}+2 T \sin \alpha \cos 1.98^{\circ}-m g \cos \gamma\right)+q \\
\dot{q}=\frac{1}{I_{y y}}\left[\begin{array}{c}
\bar{q} S \bar{c}\left(C_{M}\right)+l_{z}\left(\bar{q} S C_{L} \sin \alpha-\bar{q} S C_{D} \cos \alpha\right)+ \\
l_{x}\left(\bar{q} S C_{L} \cos \alpha+\bar{q} S C_{D} \sin \alpha\right)+l_{z e}\left(2 T \cos 1.98^{\circ}\right)
\end{array}\right] \\
\dot{\theta}=q
\end{gathered}
$$

where $m, g, \bar{q}, S, T$, and $I_{y y}$ are the mass, the gravitational acceleration, the dynamic pressure, the wing area, thrust and the inertia, respectively. Note that the thrust line is slanted about $1.98^{\circ}$ from the aircraft centre line. $C_{D}, C_{L}$, and $C_{M}$ denote the aerodynamic coefficients for drag, lift and moment. The aerodynamic coefficients are defined as follow: 


$$
\begin{gathered}
C_{D}=C_{D_{0}}+C_{D_{\delta_{h}}} \delta_{h}+\frac{\bar{c}}{2 V} C_{D_{q}} q \\
C_{L}=C_{L_{0}}+C_{L_{\delta_{h}}} \delta_{h}+\frac{\bar{c}}{2 V} C_{L_{q}} q \\
C_{M}=C_{M_{0}}+C_{M_{\delta_{h}}} \delta_{h}+\frac{\bar{c}}{2 V} C_{M_{q}} q
\end{gathered}
$$

where $\bar{c}$ is the mean aerodynamic chord. The data can be retrieved as in [22]. The small subscripts $0, \delta_{h}$, and $q$ represent the aerodynamics derivatives with respect to basic airframe, stabilator deflection and pitch rate for the respective variables.

The aircraft dynamics at longitudinal condition is always categorized into two underdamped oscillatory modes; the short period mode and the phugoid mode. In this paper, the dynamics of short period is more of interest as it is faster than the phugoid mode and it involves rapid changes to angle of attack and pitch angle.

\section{SLIDING MODE CONTROL DESIGN}

The control objective is to ensure a control law for the fighter aircraft model such that it tracks a given path for angle of attack $\alpha$ and pitch angle $\theta$ in the inertial frame with a closed loop stability. The model is assumed to be underactuated with affine system and written as follows:

$$
\begin{gathered}
\dot{\boldsymbol{x}}=\boldsymbol{F}(\boldsymbol{x})+B \boldsymbol{u} \\
\boldsymbol{y}=\boldsymbol{h}(\boldsymbol{x})
\end{gathered}
$$

The state vector is defined by $\boldsymbol{x}=[V, \alpha, q, \theta]^{T}$ which represents the velocity, angle of attack, pitch rate and pitch angle. The control vector is determined by two inputs, $\boldsymbol{u}=$ $\left[\delta_{h}, T\right]^{T}$, which represent the stabilator and thrust. Since the objective is to track angle of attack, $\alpha$, and pitch angle, $\theta$; thus $\boldsymbol{h}=[\alpha, \theta]^{T}$. Based on the outputs, by reorganizing the state variables in (8) and (9) into a matrix form, then the MIMO system can be rewritten as follows:

$$
\overbrace{\left[\begin{array}{c}
\dot{\alpha} \\
\dot{q}
\end{array}\right]}^{\dot{x}}=\overbrace{\left[\begin{array}{c}
f_{\alpha} \\
f_{q}
\end{array}\right]}^{\boldsymbol{F}(\boldsymbol{x})}+\overbrace{\left[\begin{array}{ll}
b_{11} & b_{12} \\
b_{21} & b_{22}
\end{array}\right]}^{\boldsymbol{B}} \overbrace{\left[\begin{array}{c}
\delta_{h} \\
T
\end{array}\right]}^{\boldsymbol{u}}
$$

where;

$$
f_{\alpha}=-\frac{\bar{q} S}{m V}\left(C_{L_{0}}+\frac{\bar{c}}{2 V} C_{L_{q}} q\right)-\frac{g}{V} \sin \theta \sin \alpha-\frac{g}{V} \cos \theta \cos \alpha+q
$$




$$
f_{q}=\frac{1}{I_{y y}}\left[\begin{array}{c}
\bar{q} S \bar{c}\left(C_{M_{0}}+\frac{\bar{c}}{2 V} C_{M_{q}} q\right)+ \\
\bar{q} S l_{z}\left(C_{L_{0}} \sin \alpha-C_{D_{0}} \cos \alpha\right)+ \\
\frac{\bar{q} S \bar{c} l_{z}}{2 V}\left(C_{L_{q}} \sin \alpha-C_{D_{q}} \cos \alpha\right) q+ \\
\bar{q} S l_{x}\left(C_{L_{0}} \cos \alpha+C_{D_{0}} \sin \alpha\right)+ \\
\frac{\bar{q} s \bar{c} l_{z}}{2 V}\left(C_{L_{q}} \cos \alpha+C_{D_{q}} \sin \alpha\right)
\end{array}\right]
$$

and the controller matrix is such that:

$$
\begin{gathered}
b_{11}=-\frac{\bar{q} S}{m V} C_{L_{\delta_{h}}} \\
b_{12}=\frac{2}{m V} \sin \alpha \cos 1.98^{0} \\
b_{21}=\frac{\bar{q} s}{I_{y y}}\left[\bar{c} C_{M_{\delta_{h}}}+l_{z}\left(C_{L_{\delta_{h}}} \sin \alpha-C_{D_{\delta_{h}}} \cos \alpha\right)+l_{x}\left(C_{L_{\delta_{h}}} \cos \alpha+C_{D_{\delta_{h}}} \sin \alpha\right)\right] \\
b_{22}=\frac{2}{I_{y y}} l_{z_{e}} \cos 1.98^{0}
\end{gathered}
$$

For the SMC, it is important to select an appropriate sliding surface as it plays an essential role in describing the dynamics of the system. Thus, it should be selected in consideration of the desired aircraft dynamics in which in this review; angle of attack $\alpha$ and pitch angle $\theta$ are selected. They are defined as a function of tracking error given by:

$$
\left[\begin{array}{c}
S_{\alpha} \\
s_{\theta}
\end{array}\right]=\left[\begin{array}{c}
e_{\alpha} \\
\dot{e}_{\theta}+k e_{\theta}
\end{array}\right]
$$

where $\mathrm{k}$ determines the slope of the sliding surface and can be any positive number, while $\mathrm{e}$ is the difference between output measurement and set point such as:

$$
\left[\begin{array}{l}
e_{\alpha} \\
e_{\theta}
\end{array}\right]=\left[\begin{array}{l}
\alpha-\alpha_{d} \\
\theta-\theta_{d}
\end{array}\right]
$$

The aim of the SMC is to guarantee that the outputs always follow the desired trajectory, i.e., error $e$ and its derivative $\dot{e}$ must be zero or approaching to zero as time t is increased. The derivatives of (18) then are written as:

$$
\left[\begin{array}{c}
\dot{e}_{\alpha} \\
\dot{e}_{\theta}
\end{array}\right]=\left[\begin{array}{l}
\dot{\alpha}-\dot{\alpha}_{d} \\
\dot{\theta}-\dot{\theta}_{d}
\end{array}\right]
$$

Once the sliding manifold has been defined, then the control law is designed in such a way that it drives the output to the desired value and satisfy the reachability condition leading towards an integrated SMC-MIMO control architecture. 


\subsection{Reachability Condition}

A candidate function based on the Lyapunov function is given by:

$$
V=\frac{1}{2} s^{2}
$$

Using the Lyapunov function, the derivative of the sliding surface can be written as:

$$
\dot{s} \leq-\eta \operatorname{sgn}(s)
$$

Similarly, if (17) are differentiated, then the equation is given as:

$$
\left[\begin{array}{c}
\dot{s}_{\alpha} \\
\dot{s}_{\theta}
\end{array}\right]=\left[\begin{array}{c}
\dot{\alpha}-\dot{\alpha}_{d} \\
\left(\dot{q}-\dot{q}_{d}\right)+k\left(\dot{\theta}-\dot{\theta}_{d}\right)
\end{array}\right]
$$

Using the information in (21) and substitute in (22), respectively; then:

$$
\left[\begin{array}{c}
\left(\dot{\alpha}-\dot{\alpha}_{d}\right) \\
\left(\dot{q}-\dot{q}_{d}\right)+k\left(\dot{\theta}-\dot{\theta}_{d}\right)
\end{array}\right]=\left[\begin{array}{l}
-\eta_{\alpha} \operatorname{sgn}\left(s_{\alpha}\right) \\
-\eta_{\theta} \operatorname{sgn}\left(s_{\theta}\right)
\end{array}\right]
$$

Solving for the $\dot{\alpha}$ and $\dot{q}$, and

$$
\left[\begin{array}{c}
\dot{\alpha}_{d}-\eta_{\alpha} \operatorname{sgn}\left(s_{\alpha}\right) \\
\dot{q}_{d}-k\left(\dot{\theta}-\dot{\theta}_{d}\right)-\eta_{\theta} \operatorname{sgn}\left(s_{\theta}\right)
\end{array}\right]=\left[\begin{array}{l}
f_{\alpha} \\
f_{q}
\end{array}\right]+\left[\begin{array}{ll}
b_{11} & b_{12} \\
b_{21} & b_{22}
\end{array}\right]\left[\begin{array}{c}
\delta_{h} \\
T
\end{array}\right]
$$

Solving for the controllers; the term for the equivalent controller are given as follows:

$$
\left[\begin{array}{c}
\delta_{h} \\
T
\end{array}\right]=-\left[\begin{array}{ll}
b_{11} & b_{12} \\
b_{21} & b_{22}
\end{array}\right]^{-1}\left(\left[\begin{array}{l}
f_{\alpha} \\
f_{q}
\end{array}\right]-\left[\begin{array}{c}
\dot{\alpha}_{d}-\eta_{\alpha} \operatorname{sgn}\left(s_{\alpha}\right) \\
\ddot{\theta}_{d}-k\left(\dot{\theta}-\dot{\theta}_{d}\right)-\eta_{\theta} \operatorname{sgn}\left(s_{\theta}\right)
\end{array}\right]\right)
$$

However, due to the discontinuities in the signum function, saturation function was selected to substitute in (25) to reduce the chattering problem cause by signum function, which is defined by:

$$
\operatorname{sat}\left(s_{i}, \mu\right)=\left\{\begin{array}{cc}
1 & s_{i}>\mu \\
\frac{s_{i}}{\mu} & -\mu \leq s_{i} \leq \mu \\
-1 & s_{i}<\mu
\end{array}\right.
$$

where $\mu$ is the boundary layer thickness. The control scheme for the aircraft is generally retained as illustrated in Fig. 1 to ensure its practicality. 


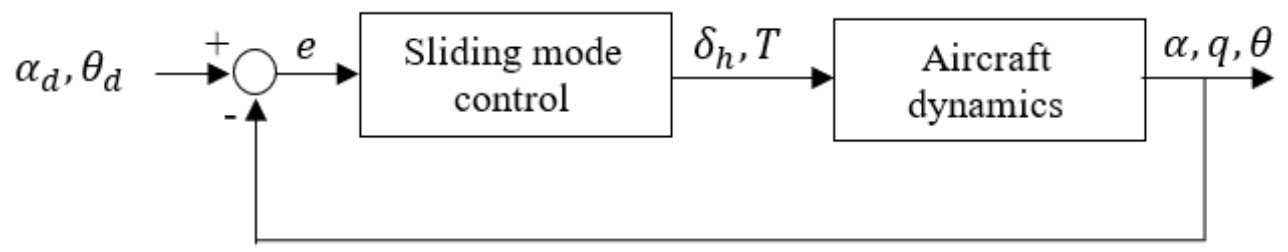

Figure 1: SMC control scheme.

\subsection{Stability Analysis}

To guarantee that the system is stabilized, the derivative of the Lyapunov function must be less than or equal to zero $(\dot{V} \leq 0)$. Thus, the stability of system in $(8)$ is analysed from the summation of the Lyapunov function in (20), which is given by:

$$
V=\frac{1}{2} s_{\alpha}^{2}+\frac{1}{2} s_{\theta}^{2}
$$

The derivative of (27) is then can be written as:

$$
\dot{V}=s_{\alpha} \dot{s}_{\alpha}+s_{\theta} \dot{s}_{\theta}
$$

From (21), (28) can be rewritten as:

$$
\dot{V}=-\eta_{\alpha}\left|s_{\alpha}\right|-\eta_{\theta}\left|s_{\theta}\right|
$$

To satisfy the inequality relation for the derivative of Lyapunov function, it can be concluded that both $\eta_{\alpha}$ and $\eta_{\theta}$ must be real positive numbers.

\section{NUMERICAL TREATMENTS}

The SMC-MIMO control architecture applied on the selected fighter aircraft model is evaluated by numerical simulations. The simulations are performed with MATLAB/Simulink to evaluate the proposed controller. The reference fighter aircraft has the following general parameters as shown in Table 1:

Table 1: Aircraft parameters [20].

\begin{tabular}{ll}
\hline Parameter & Value \\
\hline Mass, $\boldsymbol{m}(\mathrm{kg})$ & 16224.63 \\
Wing span, $\boldsymbol{b}(\mathrm{m})$ & 11.41 \\
Wing area, $\boldsymbol{S}(\mathrm{m} 2)$ & 37.16 \\
Aerodynamic centre to centre of gravity in x direction, $\boldsymbol{l}_{\boldsymbol{x}}(\mathbf{m})$ & 0.09 \\
Aerodynamic centre to centre of gravity in z direction, $\boldsymbol{l}_{\boldsymbol{z}}(\mathbf{m})$ & 0.071 \\
Engine distance, $\boldsymbol{l}_{\boldsymbol{z}_{\boldsymbol{e}}}(\mathbf{m})$ & 0.071 \\
Mean aerodynamic chord, $\overline{\boldsymbol{c}}(\mathrm{m})$ & 3.51 \\
Pitch inertia, $\boldsymbol{I}_{\boldsymbol{y} \boldsymbol{y}}(\mathrm{kg} \mathrm{m} 2)$ & 236246.3 \\
\hline
\end{tabular}




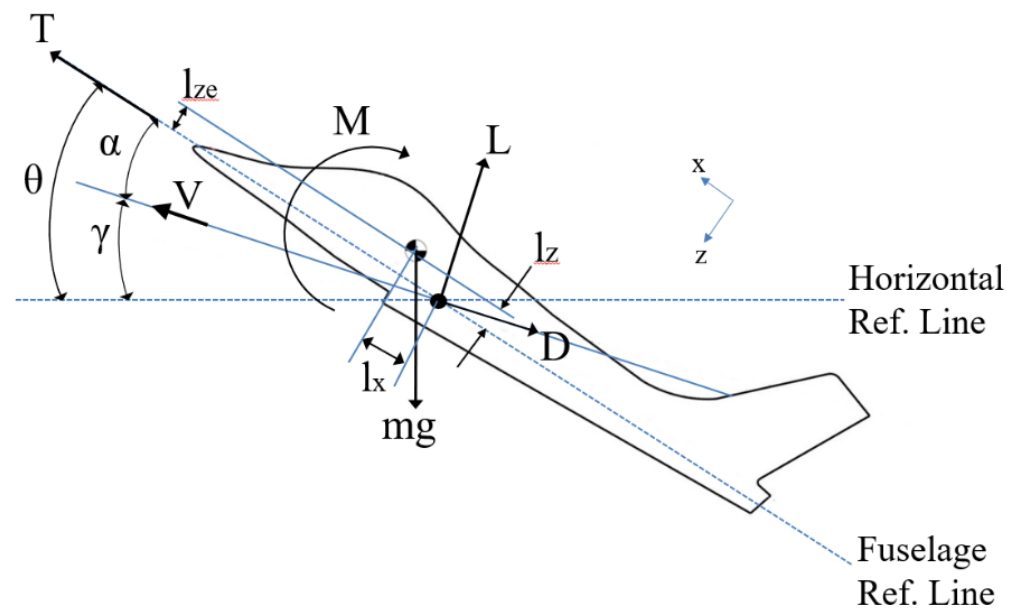

Figure 2: Aircraft loads and reference line.

The simulations were performed from a trimmed condition of velocity $V_{\text {trim }}=124 \mathrm{~m} / \mathrm{s}$, at an altitude $h_{z}=4572 \mathrm{~m}$ with the level flight condition of $\alpha_{\text {trim }}=\theta_{\text {trim }}=7.4^{\circ}$ and the inputs setting are at $\delta_{h_{\text {trim }}}=-0.078^{\circ}$ and $T_{\text {trim }}=10 \mathrm{kN}$. The numerical simulations are performed for $k=1, \eta_{\alpha}=1, \eta_{\theta}=0.3, \mu_{\alpha}=0.01$, and $\mu_{\theta}=0.01$,
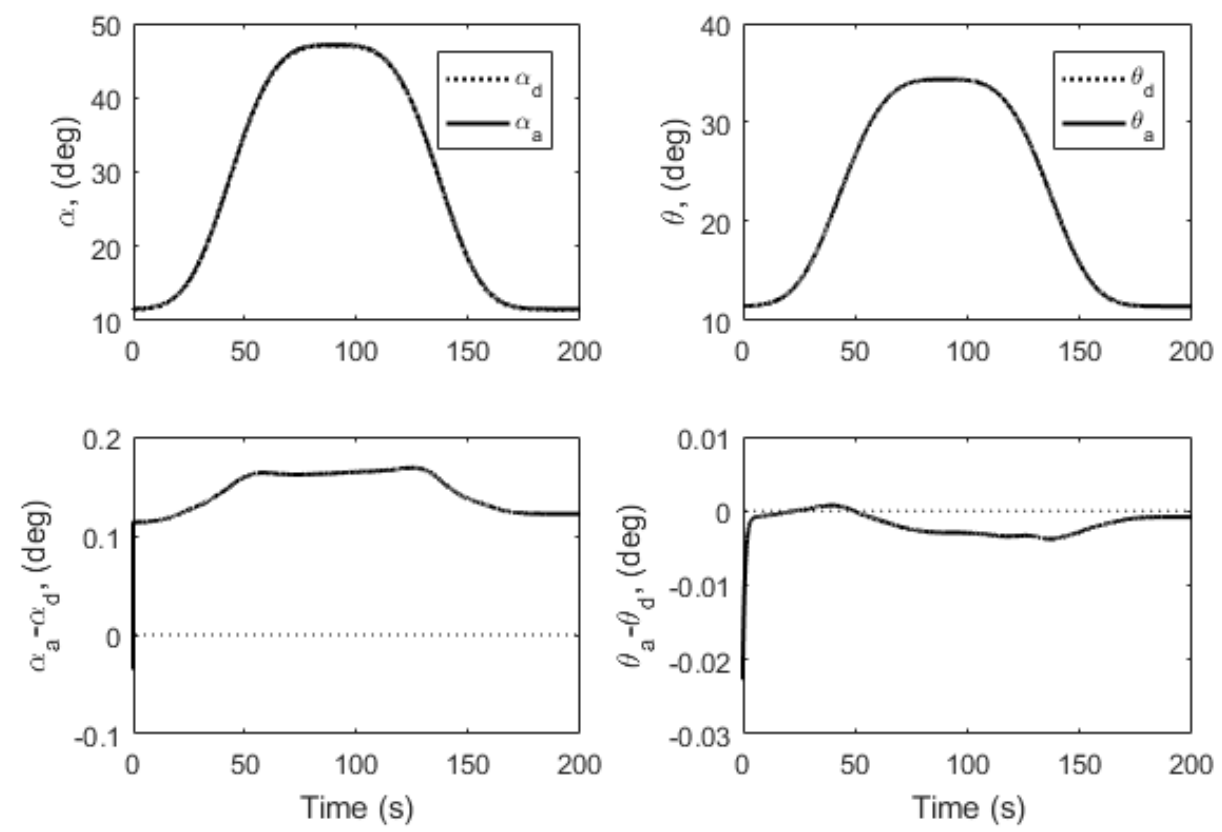

Figure 3: Angle of attack and pitch angle tracking error. 
In order to show its high $\alpha$ capabilities, a maneuver was designed to follow a demanded trajectory on $\alpha$ and $\theta$ simultaneously. The $\alpha$ and the $\theta$ are slowly increased at $\mathrm{t}=25 \mathrm{~s}$ to reach the peak values of $38 \mathrm{deg}$ for $\alpha$ and $30 \mathrm{deg}$ for the $\theta$ and slowly decrease back to their initial conditions to achieve the level flight. The time taken to complete the turns about $150 \mathrm{~s}$ for both $\alpha$ and $\theta$ as shown in Fig. 3. The demand values are designed such ways to see the effects of both state variables to the inputs setting.

The tracking errors for both state variables show a small difference and both values are converging as time increase. However, there is a residue error in $\alpha$ and $\theta$, in which the error does not conserve to 0 after certain times. Since $e_{\alpha} \neq 0$ as time increased, it may affect the $h_{z}$ as the flight path angle, defined by $\gamma=\theta-\alpha$, failed to be maintained at $0^{\circ}$. However, the condition is not that obvious as referenced to Fig. 4. This may due to the difference is considered very small which is of $0.1^{\circ}$ so the changes in $\gamma$ and $h_{z}$ are not noticeable. On the other hand, to show that the aircraft is stabilized throughout the time, a transient performance for the longitudinal response are generated as for other state variables as shown in Fig. 4.
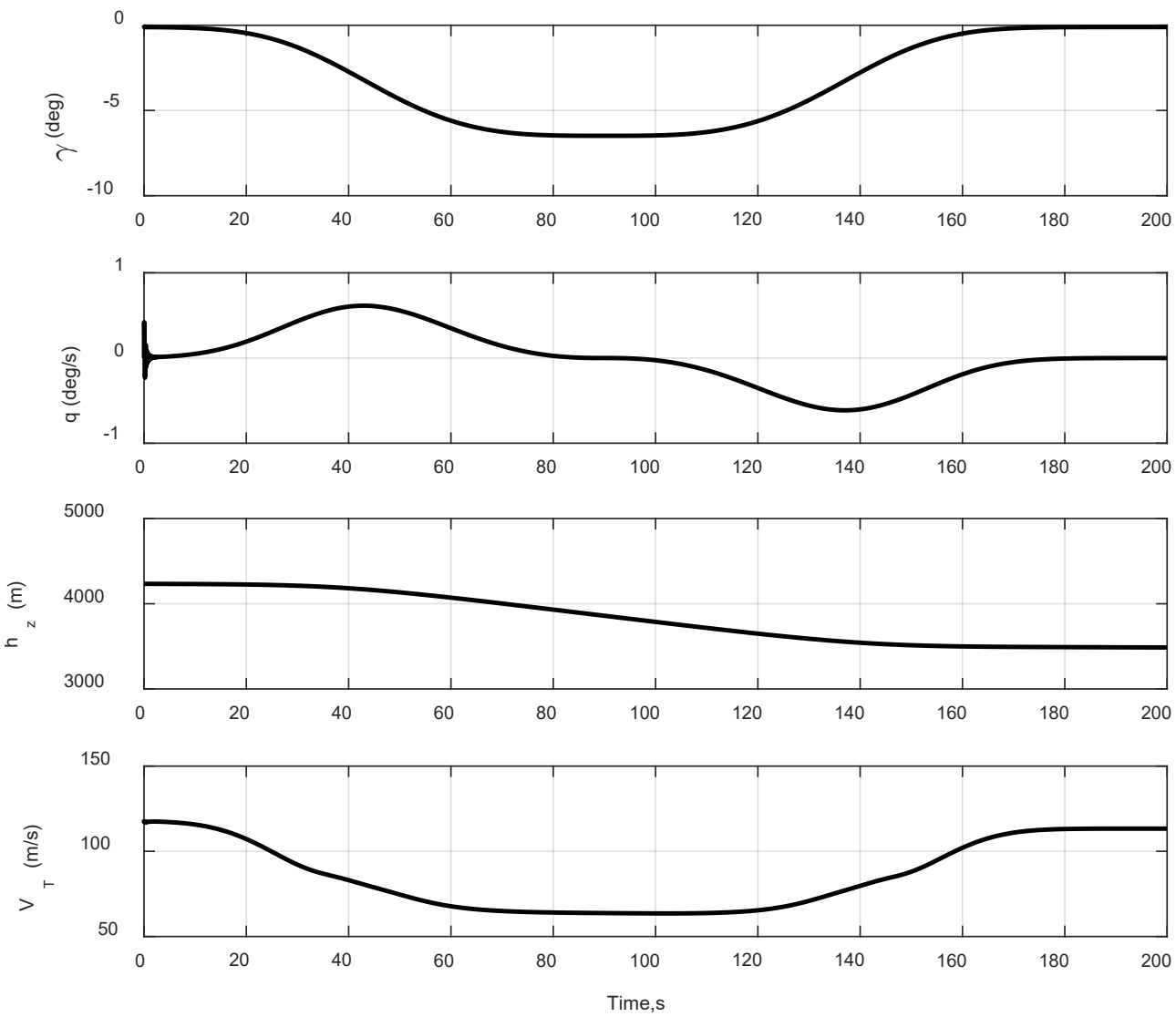

Figure 4: Longitudinal response. 
Fig. 5 shows the control efforts from the engine and stabilator as the $\alpha$ and $\theta$ are changes throughout the time. Smooth responses were expected to show the continuity function from the saturation function. However, at the beginning of the simulation time, it can be seen that the inputs response spiked to an arbitrary value due to missing information at the beginning of the time simulation at $\mathrm{t}=0 \mathrm{~s}$. This is quite typical in numerical simulations. Therefore, the results shown in Fig. 5 are acceptable; and therefore, correspond to the other results presented in Figs. 3 - 4.
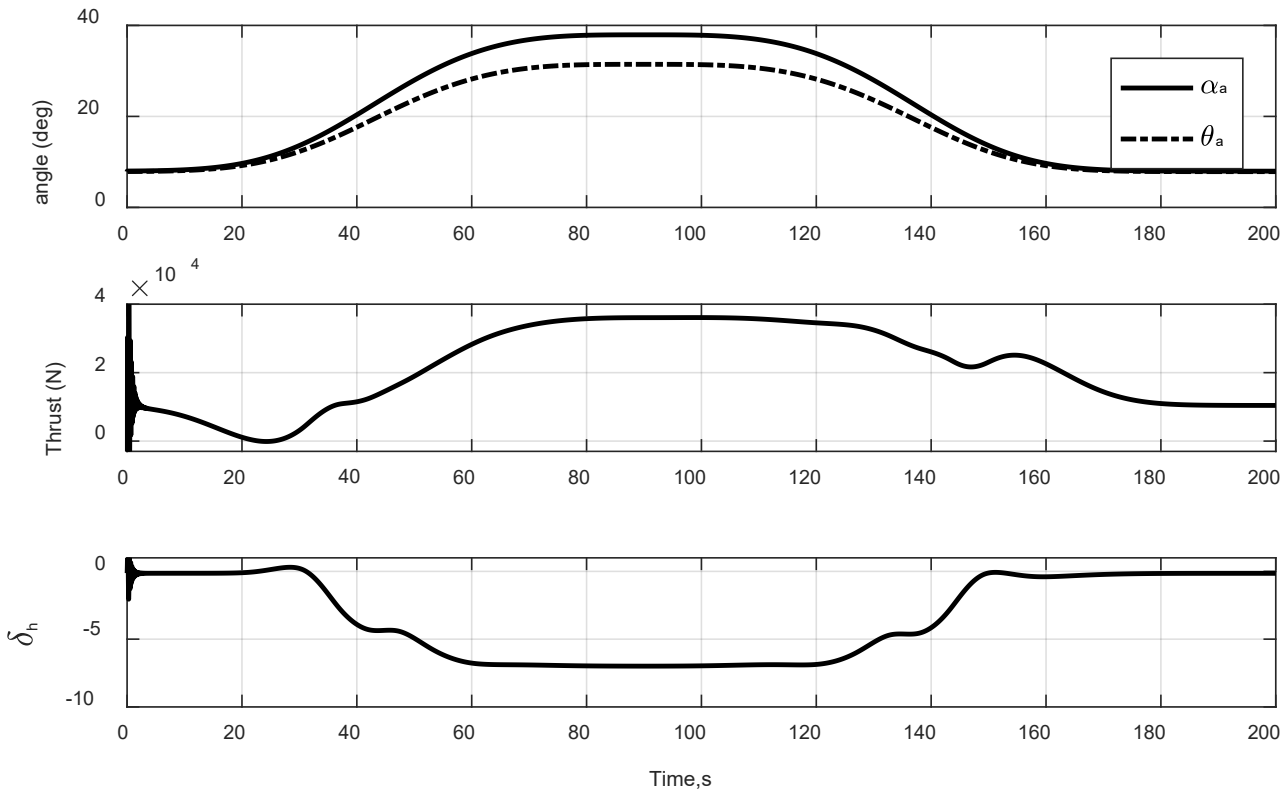

Figure 5: System input responses.

The stability of the chosen sliding surface variable of $\theta$ is shown in phase portrait plotting. Fig. 6 (a) and (b) show phase portraits with the boundary layers $\mu_{\theta}$ between 0.01 and 0.009 , which ensure the system stability and its performance.

It is noticed from Fig. 5 that the thrust $T$ almost reach 0 as the $\alpha$ is increased. Therefore, the effect of increasing $\alpha$ on the thrust setting is investigated by varying the peak value for $\alpha$ while maintaining the $\theta$ at its trimmed condition as plotted in Fig. 7. It was seen that as the peak value is increased from $15^{\circ}$ to $23^{\circ}, T$ even reached negative value while $V_{T A S}$ reached its minimum value. To make sure that the thrust keep providing a positive thrust, a configuration on the thrust is needed for example the vectored thrust, thus, to achieve a higher $\alpha$. 

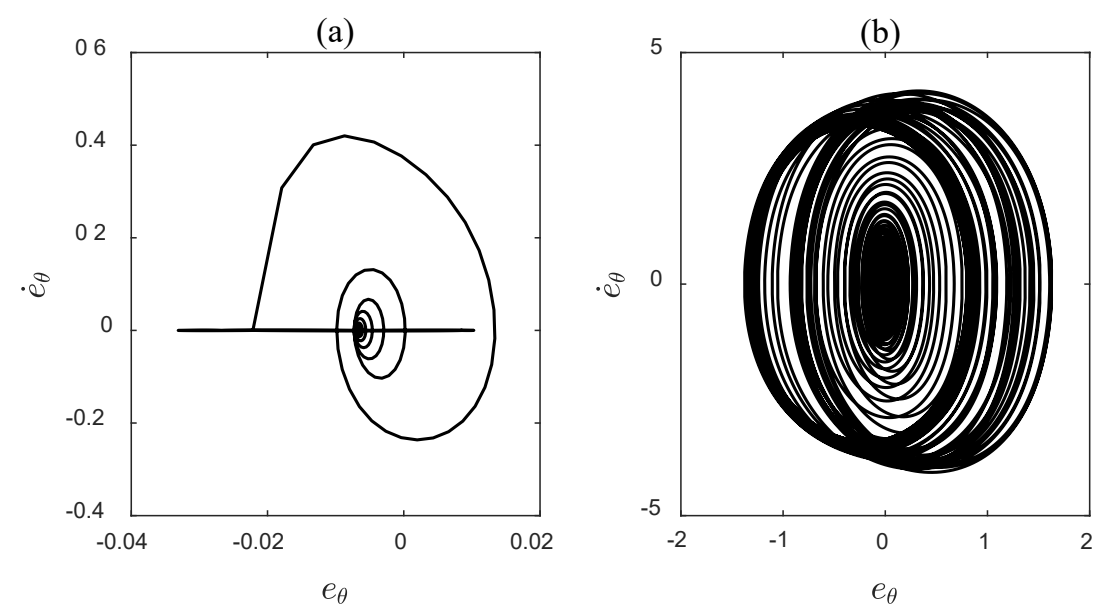

Figure 6: Phase portrait for the chosen sliding variable $\theta$ for (a) $\mu_{\theta}=0.01$ and (b) $\mu_{\theta}=0.009$.
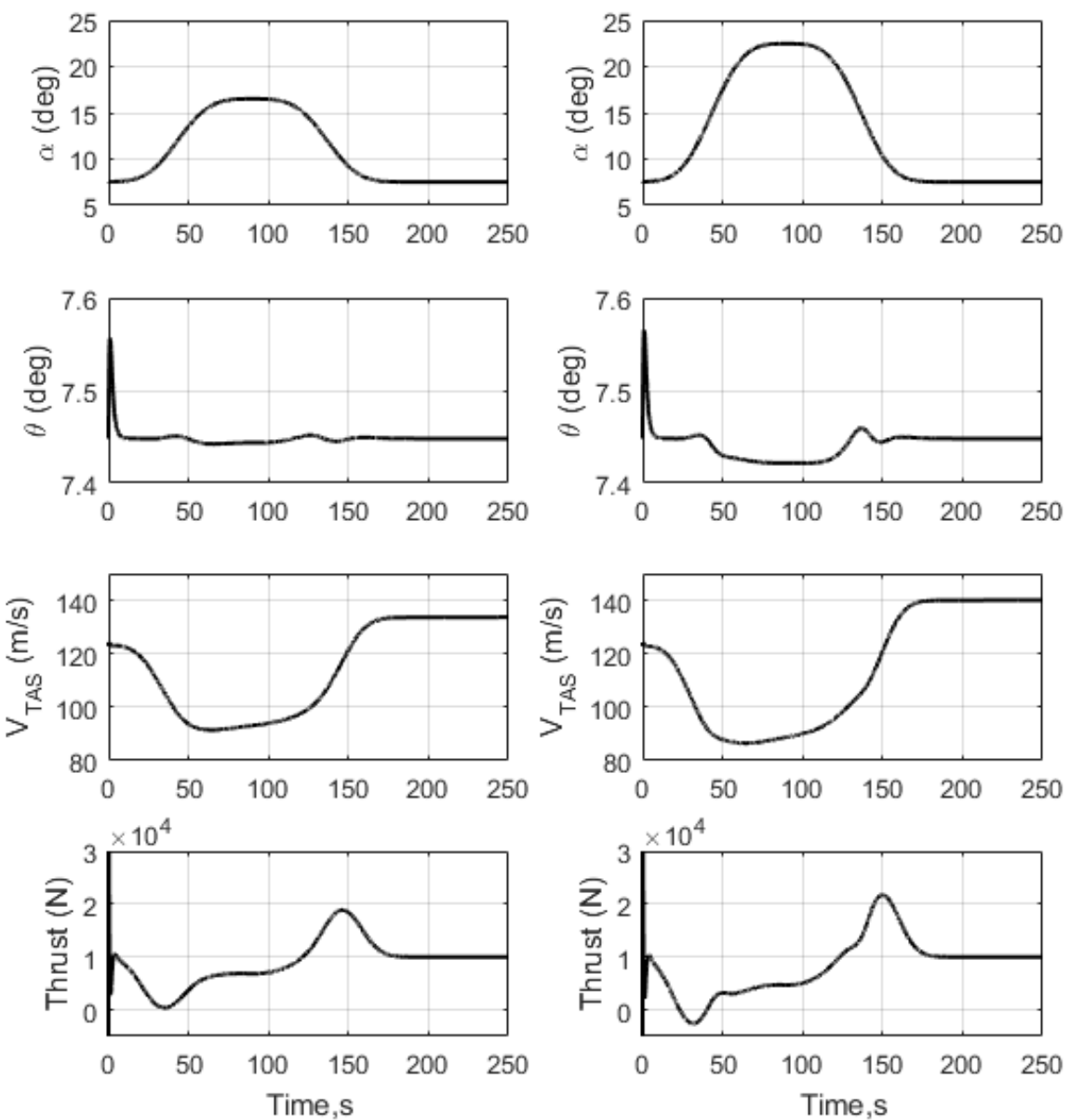

Figure 7: Effect of angle of attack on the thrust setting for (a) $\alpha_{\text {peak }}=15^{\circ}$, and (b) $\alpha_{\text {peak }}=23^{\circ}$. 


\section{CONCLUSIONS}

This feasibility study attempts to analyse the novel SMC-MIMO system in achieving high $\alpha$ in the longitudinal flight with 2 inputs, which are the stabilator and thrust. In order to handle the 2 inputs, 2 sliding surfaces are introduced for the sliding mode control. The result has shown a promising performance with an acceptable transient response; and therefore, the novel SMC-MIMO architecture is indeed a potential flight control option. In fact, different thrust configurations may further allow a higher $\alpha$ to be achieved if needed. Finally, a future work may be considered on differentiating the state variables for tracking purposes to understand the effect of the chosen state variables towards the dynamics of the high $\alpha$ apart of considering the typical vectored thrust option.

\section{REFERENCES}

[1] D. J. Stilwell, "State-Space Interpolation for a Gain-Scheduled Autopilot," Journal of Guidance, Control, and Dynamics (vol. 24, no. 3), pp. 460-465, May 2001.

[2] Z. Y. Kung and I. F. Nusyirwan, "Review on Aircraft Gain Scheduling," Journal of Advanced Review on Scientific Research (vol. 17, no. 1), pp. 1-7, 2016.

[3] S. A. Snell, W. L. Garrard, and D. F. Enns, "Nonlinear inversion flight control for a supermaneuverable aircraft," Journal of Guidance Control and Dynamics (vol. 15, no. 4), pp. 976-984, 1990.

[4] D. J. Bugajski and D. F. Enns, "Nonlinear control law with application to high angle-ofattack flight," Journal of Guidance, Control, and Dynamics (vol. 15, no. 3), pp. 761-767, 1992.

[5] G. Ramamurthi, B. Bandyopadhyay, H. Arya, and G. K. Singh, "Tracking control for nonminimum phase MIMO micro aerial vehicle - a dynamic sliding manifold approach," Proceedings of the Institution of Mechanical Engineers, Part I: Journal of Systems and Control Engineering (vol. 230, no. 9), pp. 1001-1029, 2016.

[6] Richard J. Adams James M. Buffmgton and S. S. Banda, "Design of Nonlinear Control Laws for High-Angle-of-Attack Flight," Journal of Guidance, Control, and Dynamics (vol. 17, no. 4), pp. 737-746, 1994.

[7] J. Reiner, G. J. Balas, and W. L. Garrard, "Robust Dynamic Inversion for Control of Highly Maneuverable Aircraft," Journal of Guidance, Control, and Dynamics (vol. 18, no. 1), pp. 18-24, Jan. 1995.

[8] S. A. Snell and P. W. Stout, "Robust Longitudinal Control Design Using Dynamic Inversion and Quantitative Feedback Theory," Journal of Guidance, Control, and Dynamics (vol. 20, no. 5), pp. 933-940, 1997.

[9] Q. Wang and R. F. Stengel, "Robust nonlinear flight control of a high-performance aircraft," IEEE Transactions on Control Systems Technology (vol. 13, no. 1), pp. 15-26, 2005.

[10] S. Seshagiri and H. K. Khalil, "Robust output feedback regulation of minimum-phase nonlinear systems using conditional integrators," Automatica (vol. 41, no. 1), pp. 43-54, 2005.

[11] S. Seshagiri and E. Promtun, "Sliding mode control of F-16 longitudinal dynamics," Proceedings of the American Control Conference, pp. 1770-1775, 2008.

[12]E. Promtun and S. Seshagiri, "Sliding Mode Control of Pitch-Rate of an F-16 Aircraft," IFAC Proceedings (vol. 41, no. 2), pp. 1099-1104, 2008.

[13] S. Eshghi and R. Varatharajoo, "Sliding Mode Control Techniques for Combined Energy and Attitude Control System," Applied Mechanics and Materials, vol. 629, pp. 310-317, 2014. 
[14]E. Devaud, H. Siguerdidjane, and S. Font, "Some control strategies for a high-angle-ofattack missile autopilot," Control Engineering Practice (vol. 8, no. 8), pp. 885-892, 2000.

[15]A. Das, R. Das, S. Mukhopadhyay, and A. Patra, "Sliding mode controller along with feedback linearization for a nonlinear missile model," in International Symposium on Systems and Control in Aerospace and Astronautics, 2006, no. 6, pp. 952-956.

[16]H. Vo and S. Seshagiri, "Robust Control of F-16 Lateral Dynamics," Annual Conference on IEEE Industrial Electronics, pp. 80-85, 2008.

[17] V. Rajeswari and L. Padma Suresh, "Design and Control of Lateral Axis of Aircraft using Sliding Mode Control Methodology," Indian Journal of Science and Technology, (vol. 8, no. 24), 2015.

[18]G. Guglieri and D. Sartori, "Design of a Sliding Mode Control for Wing Rock Suppression in Highly-Swept Wing Aircraft," International Journal of Aerospace Sciences, (vol. 2, no. 1), pp. 1-10, 2013.

[19] D. Shin, G. Moon, and Y. Kim, "Design of Reconfigurable Flight Control System Using Adaptive Sliding Mode Control: Actuator Fault," Proceedings of the Institution of Mechanical Engineers, Part G: Journal of Aerospace Engineering (vol. 219, no. 4), pp. 321-328, 2005.

[20] C. S. Buttrill, P. D. Arbuckle, and K. D. Hoffler, "Simulation Model of a Twin-Tail, High Performance Airplane," Hampton, Virginia, 1992.

[21] M. V. Cook, Fligth Dynamics Principle: a linear systems approach to aircraft stability and control. Butterworth-Heinemann, 2012.

[22] Documentation for a dynamic inversion control law proposed for $\mathrm{rfcs}$. http://www.nasa.gov/centers/dryden/history/pastprojects/HARV/Work/NASA2/nasa2.ht ml. Last retrieved: October 2019. 